\title{
Are the exotic mesons and baryons, recently observed, a signature of quark-hadron duality ?
}

\author{
Boris Tatischeff*
}

Institut de Physique Nucléaire, CNRS/IN2P3, F-91406 Orsay Cedex, France

\begin{abstract}
Narrow low mass exotic hadronic structures were recently observed in mesons, baryons and dibaryons. Narrow mesons, in the mass range $300 \leq \mathrm{M} \leq 750 \mathrm{MeV}$, were observed using the $\mathrm{pp} \rightarrow \mathrm{ppX}$ reaction. Narrow baryons in the mass range $1000 \leq \mathrm{M} \leq 1400 \mathrm{MeV}$ were observed using the $\mathrm{pp} \rightarrow \mathrm{p} \pi^{+} \mathrm{X}$ and $\mathrm{dp} \rightarrow \mathrm{ppX}$ reactions. The statistical significances of these structures vary up to 4.6 standard deviations (S.D.) for mesons and up to 16.9 S.D. for baryons. These exotic states are associated with precursor quark deconfinement.
\end{abstract}

\section{INTRODUCTION}

It is often believed than the observation of quark degrees of freedom cannot start at energies lower than several $\mathrm{GeV}(\approx 10 \mathrm{GeV})$. However such limit is somewhat unprecise. A relation was proposed by Baldin [1] some years ago. In this theory, the deconfinement is determined as being a process allowing particle creation for $b_{i j} \geq 5$, where

$$
b_{i j}=-\left(P_{i} / m_{i}-P_{j} / m_{j}\right)^{2}
$$

$\mathrm{P}_{i}, \mathrm{P}_{j}$ are the four momentum of both particles involved in the reaction, and $\mathrm{m}_{i}$, $\mathrm{m}_{j}$ their masses. If we consider a $\mathrm{N}-\mathrm{N}$ reaction, the previous relation reduces in the laboratory system to $\mathrm{T}_{i} \geq 2.35 \mathrm{GeV}$. Following the previous relation, the search for possible signatures of the beginning of the quark deconfinement, could be significant at energies close to $2 \mathrm{GeV}$. These correspond to the energies of the experiments which results are presented in this paper.

The measurements were performed at Saturne, with proton and deuteron beams. Their energies varied from 1.52 up to $2.1 \mathrm{GeV}$. The large momentum range SPES3 spectrometer $(600 \leq \mathrm{pc} \leq 1400 \mathrm{MeV})$ and its detection were settled at different angles from $0^{0}$ up to $17^{0}$ (lab.). Two positively charged particles ( $\mathrm{pp}$ or $\mathrm{p} \pi^{+}$) were detected and identified in the same detection. A MIT type drift chamber was located in the focal plane of the spectrometer $\left(\sigma_{x}=90 \mu \mathrm{m}\right),\left(\sigma_{\theta}=18 \mathrm{mrd}\right)$. Its efficiency was smooth, without displaying any structure. Two multidrift CERN

*e-mail: tati@ipno.in2p3.fr 
type drift chambers were located perpendicular to the mean particle trajectories. Each of these two chambers consisted of three wire planes $(\mathrm{x}, \mathrm{u}, \mathrm{v})$. The trigger consisted of four planes of plastic scintillator hodoscopes (20 scintillator/plane). A first time of flight between both extreme trigger planes were used to identify the detected particles. The time resolution for each scintillator was $\sigma \approx 180 \mathrm{ps}$. A second time of flight between both particles overdetermined the reaction under study. For $\mathrm{T}_{p}=1.52 \mathrm{GeV}$ protons at $0^{0}$, the events from the $\mathrm{pp} \rightarrow \mathrm{p} \pi^{+} \mathrm{n}$ reaction came across all the momentum range in the $\mathrm{p}_{p}$ versus $\mathrm{p}_{\pi}$ scatter plot, and the missing mass histogram was smooth without structure.

The $\sigma$ of the invariant masses was close to $1 \mathrm{MeV}$, when the $\sigma$ of the missing masses was close to $2 \mathrm{MeV}$ at forward angles.

Regular empty target measurements allowed us to observe that the target windows were not a source of noticeable contamination. They also allowed us to conclude that the data were not contaminated by any hot area of incident beam which could have been scattered by some mechanical piece at the entrance of the spectrometer. A simulation code was written in order to perform the corrections for the lost events and for the acceptance. It showed that we did not have any narrow structure in the simulated histograms, and that we have a good control of both the spectrometer and the detection. The simulation code was also used to check that the structures were not produced by eventual particles emitted vertically outside the solid angle and partially absorbed by the lead diaphragm and other mechanical pieces between the target and the spectrometer. The randoms histograms - a few $\%$ of the total spectra - did not exhibit any structure. We conclude on the lack of possible contamination, and on the genuine existence of the small and narrow observed structures.

\section{NARROW MESONS}

The missing mass of the $\mathrm{pp} \rightarrow \mathrm{ppX}$ reaction was studied at $\mathrm{T}_{p}=1.52,1.805$ and $2.1 \mathrm{MeV}$. A broad enhancement was observed between 300 and $500 \mathrm{MeV}$, which is usually called the ABC effect. The corresponding histograms displayed an oscillatory pattern, compatible with the presence of several unresolved structures [2]. The position of the extracted peaks did not depend on the multipion phase space, which exhibits a smooth behaviour. Four peaks were extracted at 313, 353, 426 and $495 \mathrm{MeV}$. When different incident proton energies and angles were considered, the stability of the two first masses was better than the stability of the two last masses.

Between the $\eta$ and the $\omega$ mesons, narrow structures were observed at 588, 608, $647,681,700,(715)^{1}$, and $(750) \mathrm{MeV}[3]$.

There is no room for these new mesons within $q \bar{q}$ models. Indeed there is an excellent agreement between the complete classical meson spectrum and the calculations using a relativistic quark model for mesons [4]. Therefore more complex quark configurations are to be considered. We call these states: exotic mesons.

\footnotetext{
${ }^{1}$ between parenthesis since its existence is less well defined
} 
Figure 1 shows an attempt to describe the experimental masses using the following phenomenological mass formula [5] for two coloured constituent quark cluster configurations:

$$
M=M_{0}+M_{1}\left[i_{1}\left(i_{1}+1\right)+i_{2}\left(i_{2}+1\right)+(1 / 3) s_{1}\left(s_{1}+1\right)+(1 / 3) s_{2}\left(s_{2}+1\right)\right],
$$

where $M_{0}$ and $M_{1}$ are parameters and $i_{1}\left(i_{2}\right), s_{1}\left(s_{2}\right)$ are isospin and spin of the first and second quark clusters respectively.

The first four meson masses, extracted from the ABC broad bump, are well described using the $q^{2}-\bar{q}^{2}$ configurations. These states are "broad" ( $\sigma$ (width) $\approx 20$ $\mathrm{MeV}$ ) and highly excited. The mesons between 480 and $620 \mathrm{MeV}$ which correspond to narrow and weakly excited peaks, are described using the $q^{3}-\bar{q}^{3}$ configurations. In both cases $\left(q^{2}-\bar{q}^{2}\right.$ and $q^{3}-\bar{q}^{3}$ configurations) the same parameters: $\mathrm{M}_{0}=310$ $\mathrm{MeV}$ and $\mathrm{M}_{1}=30 \mathrm{MeV}$ are used. Finally the mass range between 620 and $750 \mathrm{MeV}$ is described by $q^{4}-\bar{q}^{4}$ configurations with slightly different parameters (by 10\%) : $\mathrm{M}_{0}=357 \mathrm{MeV}$ and $\mathrm{M}_{1}=27 \mathrm{MeV}$.

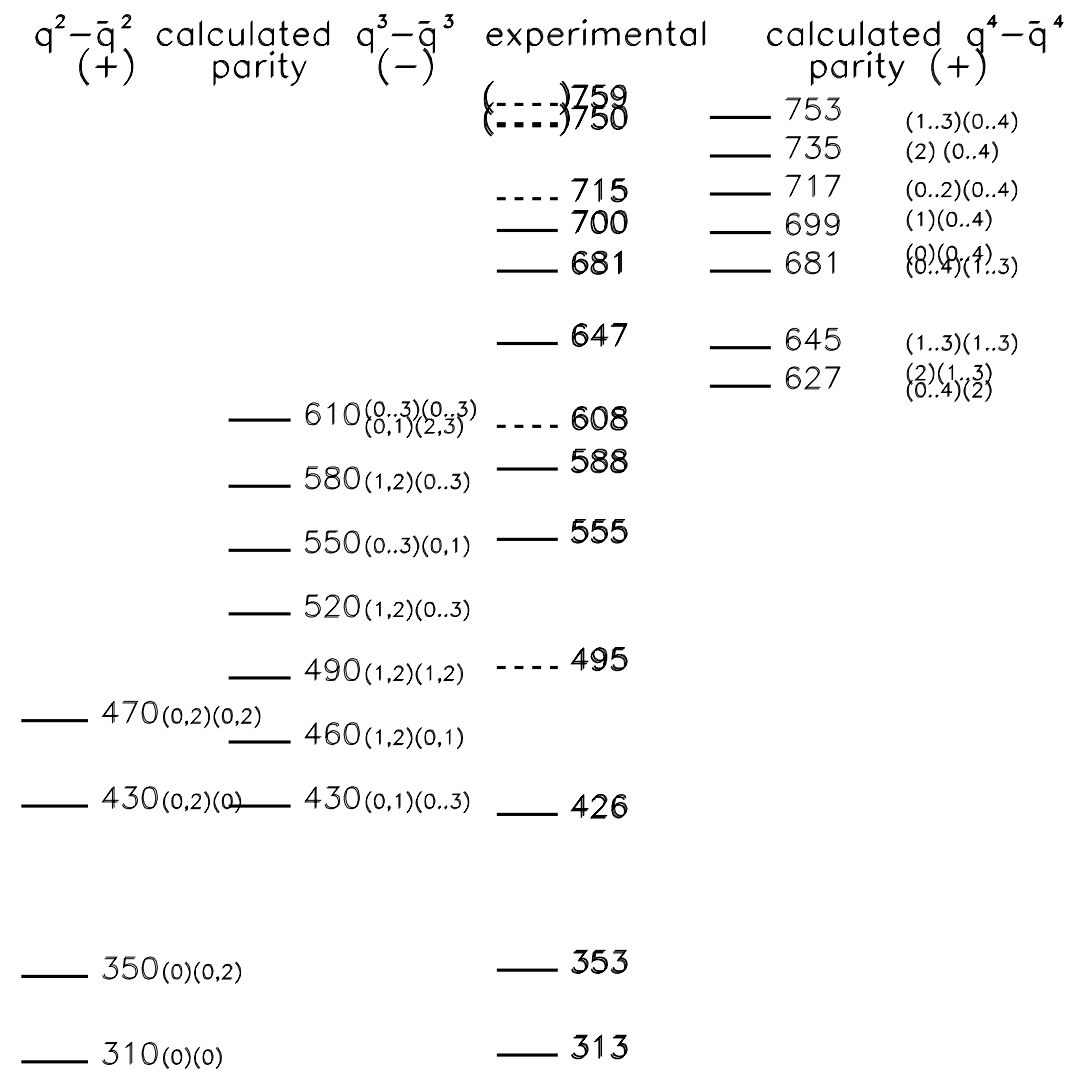

FIGURE 1. Compared experimental and calculated exotic narrow mesonic mass spectra. Into parenthesis, calculated possible (Spin) and (Isospin) respectively. 


\section{NARROW BARYONS}

The first results of narrow exotic low mass baryons were obtained using the missing mass $\mathrm{M}_{X}$ of the $\mathrm{pp} \rightarrow \mathrm{p} \pi^{+} \mathrm{X}$ reaction [6]. Three baryons were observed, with S.D. as large as 10, at 1004, 1044 and $1094 \mathrm{MeV}$. The corresponding structures were observed at nearly all incident proton energies and scattering angles.

Other baryons - which are less highly excited - were also recently extracted from the same reaction at the following masses: 1136, 1173, 1249, 1277, and (1384) MeV. Since they were each observed in only some experimental spectra corresponding to different angle and-or incident proton energy, their existence is less firmly established than was the existence of the first three lowest narrow baryons. In the mass range $1.1 \leq \mathrm{M} \leq 1.45 \mathrm{GeV}$ they were observed in the missing mass $\mathrm{M}_{X}$ or-and in the invariant mass $\mathrm{M}_{p \pi^{+}}$of the previous reaction. They were also observed in the missing mass $\mathrm{M}_{X}$ of the $\mathrm{dp} \rightarrow \mathrm{ppX}$ reaction, although with smaller S.D. These last results cannot be used to establish firmly the existence of these states. But since the masses of the structures observed in the $\mathrm{dp} \rightarrow \mathrm{ppX}$ experiment lie close to those found in the first reactions, these results seem to confirm the previous ones. Figure 2 illustrates some of these peak extractions and table 1 describes the conditions of each histogram.
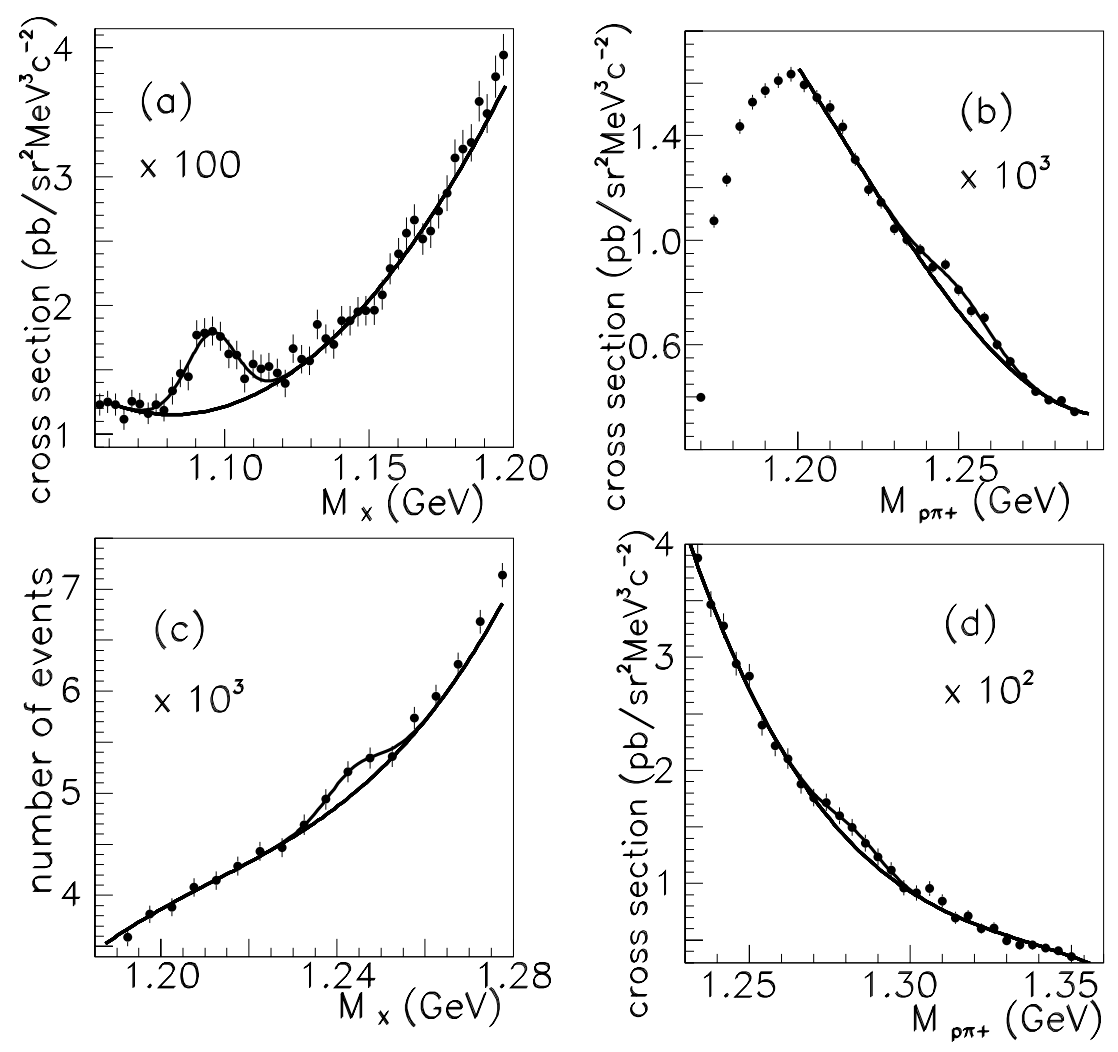

FIGURE 2. A selection of four experimental spectra, showing small and narrow baryonic structures. 


\begin{tabular}{ccccccc}
\hline \hline & Mass $(\mathrm{MeV})$ & S.D. & $\mathrm{T}_{p}(\mathrm{MeV})$ & $\theta^{0}$ & reaction & observable \\
(a) & 1094 & 10.9 & 1805 & 3.7 & $\mathrm{pp} \rightarrow \mathrm{p} \pi^{+} \mathrm{X}$ & $\mathrm{M}_{X}$ \\
(b) & 1249 & 8.3 & 1520 & 2 & $\mathrm{pp} \rightarrow \mathrm{p} \pi^{+} \mathrm{n}$ & $\mathrm{M}_{p \pi+}$ \\
(c) & 1249 & 3.2 & 2100 & 17 & $\mathrm{dp} \rightarrow \mathrm{ppX}$ & $\mathrm{M}_{X}$ \\
(d) & 1277 & 3.5 & 1805 & 6.7 & $\mathrm{pp} \rightarrow \mathrm{p} \pi^{+} \mathrm{X}$ & $\mathrm{M}_{p \pi+}$ \\
\hline \hline
\end{tabular}

TABLE 1. Description of the data of Figure 2 showing new structures as observed in different reactions, different observables, incident energies and spectrometer angles.

Only those structures extracted several times at masses less than $\mathrm{M} \pm 5 \mathrm{MeV}$ were taken into account. The widths of the structures - about a few $\mathrm{MeV}$ - are not precise enough to discriminate the experimental from the physical widths.

As it was the case for mesons, there is no room for new baryons in this mass range within the many quark models [7]. Missing baryons are baryons which were calculated by different constituent quarks models, but which were not seen experimentally through elastic $\pi \mathrm{N}$ scattering. They are predicted for masses larger than $1700 \mathrm{MeV}$, and not in the mass range studied here. Moreover there is - a priori no reason for them to be narrow since they are calculated within the three quarks assumption. We have therefore several reasons not to associate our narrow baryons to missing baryons, but to associate them to an exotic (not $\mathrm{q}^{3}$ ) origin. We considered the same phenomenological mass formula as the one already used for mesons. The two parameters were adjusted in order to get the mass, and different possible (because of large degenerascy) spin and isospin values of nucleon and Roper resonance at $1440 \mathrm{MeV}$. We got the calculated spectra shown in Figure 3 and observed a very good agreement between measured and calculated masses - mainly for the four lower baryons - although no adjustable parameter was used here.

\section{DISCUSSION}

Thanks to good resolution and statistics, narrow structures were observed in hadrons using the $\mathrm{pp} \rightarrow \mathrm{p} \pi^{+} \mathrm{X}, \mathrm{pp} \rightarrow \mathrm{ppX}$ and $\mathrm{dp} \rightarrow \mathrm{ppX}$ reactions. Many checks were performed to make sure that these structures were not produced by artefacts. Their small width and their mass stability, whatever the experiment, were used to conclude that they are genuine hadronic structures which were not produced by dynamical rescatterings. Since they cannot be associated to classical quark configurations: $q \bar{q}$ for mesons and $q^{3}$ for baryons, we tentatively associated them to coloured cluster quark configurations. The agreement between measured and calculated masses is outstanding since both spectra were obtained quite close although only two (zero) free parameters are used for mesons (baryons). It remains that our attempt to identify the experimental narrow structures to partial quark deconfinement, in these experiments of low temperature and small baryochemical potential (low baryonic number density), although being quite impressive, is not understood today and needs a theoretical explanation. 


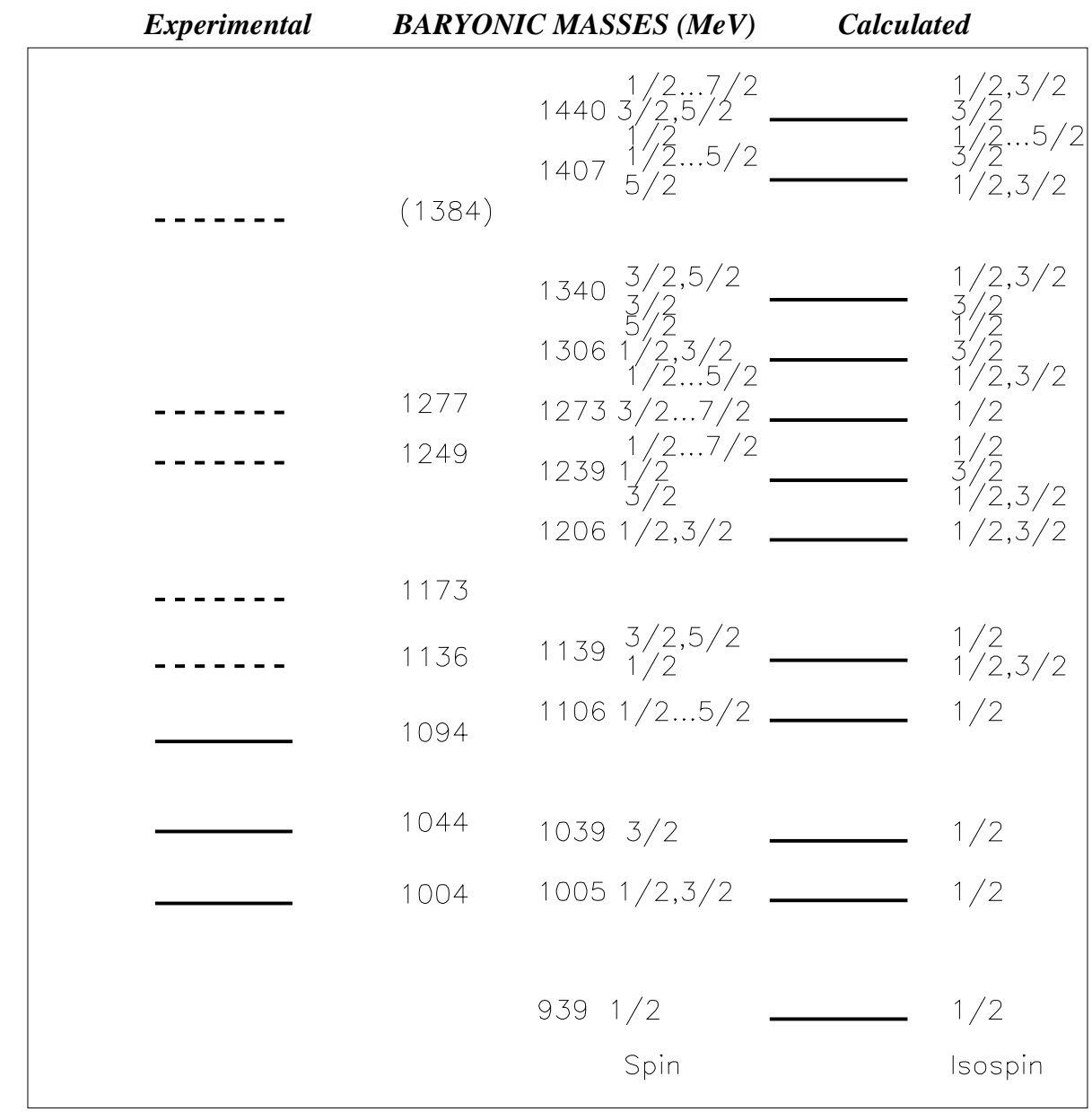

FIGURE 3. Compared experimental and calculated masses of exotic narrow baryonic structures.

\section{REFERENCES}

1. Baldin, A.M., Dubna 1992 JINR, E1-92-487.

2. Yonnet, J., Tatischeff, B., et al., Phys. Rev. C63, 014001 (2000).

3. Tatischeff, B., et al., Phys. Rev. C62, 054001 (2000).

4. Koll, M., et al., Eur. Phys. J. A9, 73 (2000).

5. Mulders, P.J., Aerts, A.T., de Swart,J.J., Phys. Rev. D21 (1980) 2653; Phys. Rev. D19 (1979) 2635; Phys. Rev. Lett. 40 (1978) 1543.

6. Tatischeff, B. et al., Phys. Rev. Lett. 79, 601 (1997).

7. Capstick, S. and Roberts, W., Progress in Particle and Nuclear Physics 45, S241 (2000). 